\title{
Téoros
}

Revue de recherche en tourisme

\section{Le caractère touristique des institutions patrimoniales et muséales}

\section{Suzanne Chassé et Jean-Paul Thomin}

Volume 11, numéro 2, juillet 1992

Quand les musées s’ouvrent au tourisme...

URI : https://id.erudit.org/iderudit/1078085ar

DOI : https://doi.org/10.7202/1078085ar

Aller au sommaire du numéro

Éditeur(s)

Université du Québec à Montréal

ISSN

0712-8657 (imprimé)

1923-2705 (numérique)

Découvrir la revue

Citer cet article

Chassé, S. \& Thomin, J.-P. (1992). Le caractère touristique des institutions patrimoniales et muséales. Téoros, 11(2), 7-12.

https://doi.org/10.7202/1078085ar d'utilisation que vous pouvez consulter en ligne.

https://apropos.erudit.org/fr/usagers/politique-dutilisation/ 


\title{
Le caractère touristique des institutions patrimoniales et muséales
}

\author{
Suzanne Chassé et Jean-Paul Thomin*
}

Afin de micux connaitre le caractère touristique des musces, nous allons, dans un premier temps, traiter des publics en analysant la motivation de ces demiers à visiter ces institutions et en identifiant parmi eux, la clientěle touristique. Dans un second temps, nous allons définir comment l'institution musçale peut être un produit touristique tant dans son approche muséale traditionnelle que dans son approche interpretative et le degré d'attractivité de ces équipements pour les touristes. Troisiemement, nous évaluerons la mise en marché touristique actuellede ces institutions culturelles dans le produit touristique québécois et nous conclurons sur le devenir touristique de ces dernieres.

\section{L'évaluation des publics}

Afin de mieux connaître les différents publics des musées, nous analyserons, dans un premier temps, deux typologies de clientèles et en second lieu, nous identifierons l' importance de la clientele touristique dans la fréquentation des institutions muséales du Québec.

\section{Typologie des visiteurs}

Plusieurs typologies concemant les publics des musćes ont été élaborées par des Américains ou par des Européens (français, britannique, etc.). Afinde mieux cemer la clientèle touristique, nous retiendrons la typologie développée aux États-Unis par Marylin Hood ${ }^{(1)}$ pour sa these de doctorat et celle effectuée pour la direction des musées de France par Hana Gottesdiener et Jean Dava$\operatorname{lon}^{2}$. La premiere est basée sur le taux de fréquentation des visiteurs et la seconde, sur la motivation des visiteurs.

\footnotetext{
Madame Suzanne Chassé est professeure de tourisme au college de Granby et associesexpert chez Zins, Beauchesne et associés. Monsieur Jean-Paul Thomin est président de la firmeldéation-Cornmunications.
}

\section{Typologie et taw de frequentation}

Cette typologie tient compte du nombre de visites annuelles effectuces par les visiteurs dans les institutions. On répartit la clientèle en quatre groupes: les specialistes, les visiteurs ayant une frequentation annuelle de plus de trois visites, les visiteurs occasionnels (une fois l'an) et les non visiteurs.

Le comportement des Québécois pourrait se répartir de la façon suivanted9):

- spécialistes:

- visiteurs fréquents

- visiteurs occasionnels $5 \%$

- non-visiteurs

$15 \%$

$20 \%$

$60 \%$

- Le spécialiste est celui qui fréquente le musée pour son travail, ou parce qu'il est passionné par le thème développé par l'institution.

- Le visiteur fréquent est un habitué des musées, son niveau de scolarité est plus élevéet, trèsjeune, ilapris I' habitude de visiter ces institutions en famille, avec un professeur passionné ou un ami.

- Le visiteur occasionnel se regroupe en deux catégories: d'une part, celui qui visite un musée parce qu'on y présente une exposition majeure (blockbuster) qu'il se doit de voir et, d'autre part, le voyageur ou le touriste qui profite de son séjour dans un pays ou une région pour découvrir ses attraits. Les musées représentent une part importante etsouvent majeure dupotentiel at tractif d"une région.

- Le non-visiteur, quant a lui, ne considêre pas la visite d'un musée comme un loisir ou comme un moment de détente privilégié. Il préfere les activités de plein-air; le sport et les rencontres sociales. Il considère que cette visite demanderait trop d'efforts intellectuels et il est peu intéressé par l'apprentissage.

Cette première typologie identifie le touriste comme étant un visiteur occasionnel. Des groupes de discussion menés lors d'études de faisabilité pour l'implantation de musées au Québec (Musées du cuir, de l'industrie laitière, de la course automobile, etc,), nous ont permis de constater qu'en voyage, deux facteurs peuvent influencer la décision d'aller visiter un musce; d' une part, la disponibilité de temps et d'autre part, l'ouverture à la découverte dont quelque fois lesenfants sont le prétexte.

\section{Typologie et motivations des visiteurs}

La seconde typologie a été développée par Jean Davalon et Hana Gottesdiener. Celle-ci est basée sur la motivation personnelle des visiteurs. Cette motivation est double; elle peut être liée au savoir et à la connaissance, ou au champ personnel du visiteur (ses émotions ou ses intérêts).

Ils classifienten quatre catégories les visiteurs: le touriste; l'esthete; le pedagogue ou l'ethnologue; le scientifique ou le technicien.

- Le touriste et l'esthète apprócient les musées pour leur capacité d'emouvoir. $\mathrm{C}^{\prime}$ est la réaction que provoque la visite qui justifie la fréquentation. Cette réaction est en lien direct avec leurs goûts et leur histoire personnelle. Cette visite doit provoquer une émotion et c'est davantage l' agrément du décor, de l'environnement, des jardinset des salles qui crént cette satisfaction. L'experience plus contemplative de cette visite s'inscrit dans le corps plutôt que la raison. On apprécie sa visite car on la considere comme un moment dedétente et de divertissement.

De plus, les themes ayant un lien avec leur quotidien ou leur environnement plus immédiat les rejoignent davantage. Une êtude menée par madame Hana Gottesdiener sur la fréquentation des musées d'art nous révèle que ces demiers ont un public different des musces $\mathrm{d}^{4}$ histoire naturelle. Les premiers sont visités davantage par les cadres $(52 \%)$, tandis que les seconds le sont pardes ouvriers non-qualifiés ( $34 \%$ ) et employés ( $43 \%$ ). 
- Quant aux pédagogues et scientifiques, ils privilégient le savoir et c'est la capacité d'informer qu'ils jugent primordial. Ces derniers mettent beaucoup d'importance sur les objets, la valeur des collections et les messages véhiculés. Ils privilégient essentiellement le volet cognitif de leur expérience. Deplus, pour ceux-ci, une visite des institutions muséales permet une valorisation de l'image de soi car l'expérience permet d'enrichir leurs connaissances, Parmices visiteurs, certains preferentque les institutions conservent leur caractère élitiste.

Le tableau 1 qui suit fait la synthèse des deux approches.

\section{Analyse de la fréquentation des institutions muséales du Québec}

Afin de bien cerner l' importance de la clientèle touristique pour ces institutions, deux volets seront analysés; d'une part, l'achalandage en nombre absolu et d'autre part la provenance de cette clientèle.

\section{L'achalandage des musées}

A partir des données de fréquentation de 1990 que possede le ministère des Affaires culturelles $^{(4)}$, on peut répartir l'achalandage des musées qu'il subventionne, en trois classes:

- moins de 15000 visiteurs : 18 institutions - de 15000 à 50000 visiteurs: 17 institutions

- plus de 50000 visiteurs : 4 institutions

La majorité des institutions de la première classe sont d'intérêts locaux ou régionaux (Musćes Missisquoi, Kamouraska, etc.); la seconde classe regroupe les musées d'intérêtts provinciaux ou situés sur des circuits touristiques bien commercialisés (Musées d'arts décoratifs, de la Gaspésie, etc.) et les quatre institutions de la troisième classe sont des musées situésà QuébecouàMontréal, de portée internationale et bénéficiant d'un bassin de population beaucoup plus important (Musées des Beaux-Arts, de la Civilisation, etc.).

\section{Provenance des visiteurs}

Une enquêtte menée auprès des musées et centres d'exposition du Québec, nous révèle qu'approximativement, $38 \%$ des visiteurs

TARLEAU 1: Publics et institutions: une DOUBLE approche

Musée $=$ temple $=$ SAVOIR $\quad$ Musée $=$ espace public $=$ ÉMOTION

Sacralisation, élitisme Démocratisation

Public; scientifiques et

pédagogues, fréquents

Publics; touristes, occasionnels

TABLEAU 2: Provenance des visiteurs des musées québécois ${ }^{(5)}$ (1991)

\begin{tabular}{|c|c|c|c|c|}
\hline Clientèle & $\begin{array}{r}\text { Ensemble } \\
\text { du Québec }\end{array}$ & Montréal & Québec & Régions \\
\hline $\begin{array}{l}\text { Locale } \\
\text { Régionale }\end{array}$ & $\begin{array}{l}42,2 \% \\
19,9 \%\end{array}$, $62.1 \%$ & $\begin{array}{l}65,6 \% \\
11,8 \%\end{array}$ & $\begin{array}{l}43,1 \% \\
14,3 \%\end{array}$ & $\begin{array}{l}32,2 \% \\
24,5 \%\end{array}$ \\
\hline $\begin{array}{l}\text { Québécoise } \\
\text { Canadienne } \\
\text { Extérieure }\end{array}$ & $\begin{array}{r}23,3 \% \\
6,3 \% \\
8,3 \%\end{array} \mid 37.9 \%$ & $\begin{array}{l}9,2 \% \\
4,6 \% \\
8,7 \%\end{array}$ & $\begin{array}{r}22,7 \% \\
8,2 \% \\
11,8 \%\end{array}$ & $\begin{array}{r}29,6 \% \\
6,6 \% \\
7,4 \%\end{array}$ \\
\hline
\end{tabular}

sont des touristes et que, parmi ces derniers, $62 \%$ proviennent du Québec (tableau 2).

On s'aperçoit que lorsque le bassin de population est moindre, la clientèle touristique devient proportionnellement très importante dans l'achalandage des institutions; d'où l'intérêt pour les gestionnaires de bien positionner leur muséedans le produit touristique régional.

\section{La définition de l'institution patrimoniale et muséale comme produit touristique}

\section{Les composantes fondamentales}

Si on aborde le milieu muséal par son désir d'attirer le visiteur, on peut, grosso modo, identifier deux grands types dintervention : l'interprétation et la muséologie proprement dite, dont l'évolution rapide au cours des dernières années a d'ailleurs généré plusieurs nouvelles métamorphoses qui tendent depuis peu à joindre les deux secteurs au coeur d'une même pratique de l'approche des publics.
Un premier type générique d'approche des publics: l'interprétation

L'interprétation constitue théoriquement le type générique le plus tourné vers l' ensemble des publics. Au Québec, elle tire en bonne partie son origine des efforts d'animation développés par le National Service Park des États-Unis pour rendre plus accessibleả l'ensemble des publics la signification des lieux naturels et historiques. Avec le temps, la méthode interprétative s'est davantage cristallisće autour de l'exposition auto-guidée où le cheminement du visiteur est en principe orienté par le parcours visuel de l'exposition. Sur le plan conceptuel, le centre d'interprétation définit alors sa capacité à atteindre le plus large public par le dynamisme et l'efficacité pédagogique des moyens déployés par l'exposition pour communiquer aux visiteurs lesens et le contenudu site ou du thème. Le médium visuel massivement employé par les expositions est le design.

A partir des efforts entrepris par Parcs Canada pour constituer un réseau étoffé de centres d'interprétation (particulièrement entre 1979 et 1985), on s'est mis à considérer que les caractéristiques des expositions adaptées. sur le plan conceptuel, aux goûts 
des publics, pouvaient faire de ces centres un élément pratique d'attrait touristique. Les années 1985 à 1990 ont donc vu jaillir au Québec environ une vingtaine de ces centres d'interprétation, misdel'avant par une grande diversité d'organismes communautaires, et appuyés sur le désir des collectivités d'être culturellement (et parfois aussi bien socialement qu'économiquement) représentés et financés (a priori) à titre d'attraits touristiques par divers Ministères.

Suite aux résultats préliminaires obtenus au cours des dernières années, une réflexion embryonnaire a commencé sur l'efficacité attractive des centres d'interpretation pour déboucher sur un relatif éclatement du système interprétatif au profit d'une multiplication des langages visuels possibles: scénographie, environnement visuel intégrant art, scénographie et design, etc, qui parfois, par la place nouvelle faite aux collections, rapprochent davantage l'interprétation de la muséologie proprement dite.

\section{L'évolution d'un deuxième type : les musées proprement dit}

Le monde muséal a connu à son tour, au cours des dernières années, un véritable éclatement des genres qui donne priorité à la recherche de la formule harmonique entre la satisfaction des publics, la mise en valeur maximale des expositions et l'accroissement de la marge d'auto-financement (que ce soit par la justification des subventions grâce aux succès publics obtenus ou de ceux, très virtuels, enregistres aux guichets).

La définition des fonctions d'un musce telle que décrite par l'ICOM (préservation, diffusion et recherche) devient sujette aux remises en question au fur et à mesure que se modifie un contexte aux réalités à la fois mouvantes et changeantes. La collection, lorsqu"elle représente la justification de l'existence du musée (ce qui exclut alors bon nombre de musées des sciences et de sociétés comme les musées des enfants), devient l'enjeu d'un débat entre la préservation du caractère sacré du musée et l'utilisation optimum d'un outil unique et original d'attrait des publics.

Le Musée de la civilisation à Québec représente un très bon exemple d'une institution enlignée en vue d'une adaptabilité optimum aux conditions changeantes du contexte culturel:

- l'utilisation intensive de la scénographie (Souffrir pour être belle, Mi-wrai'

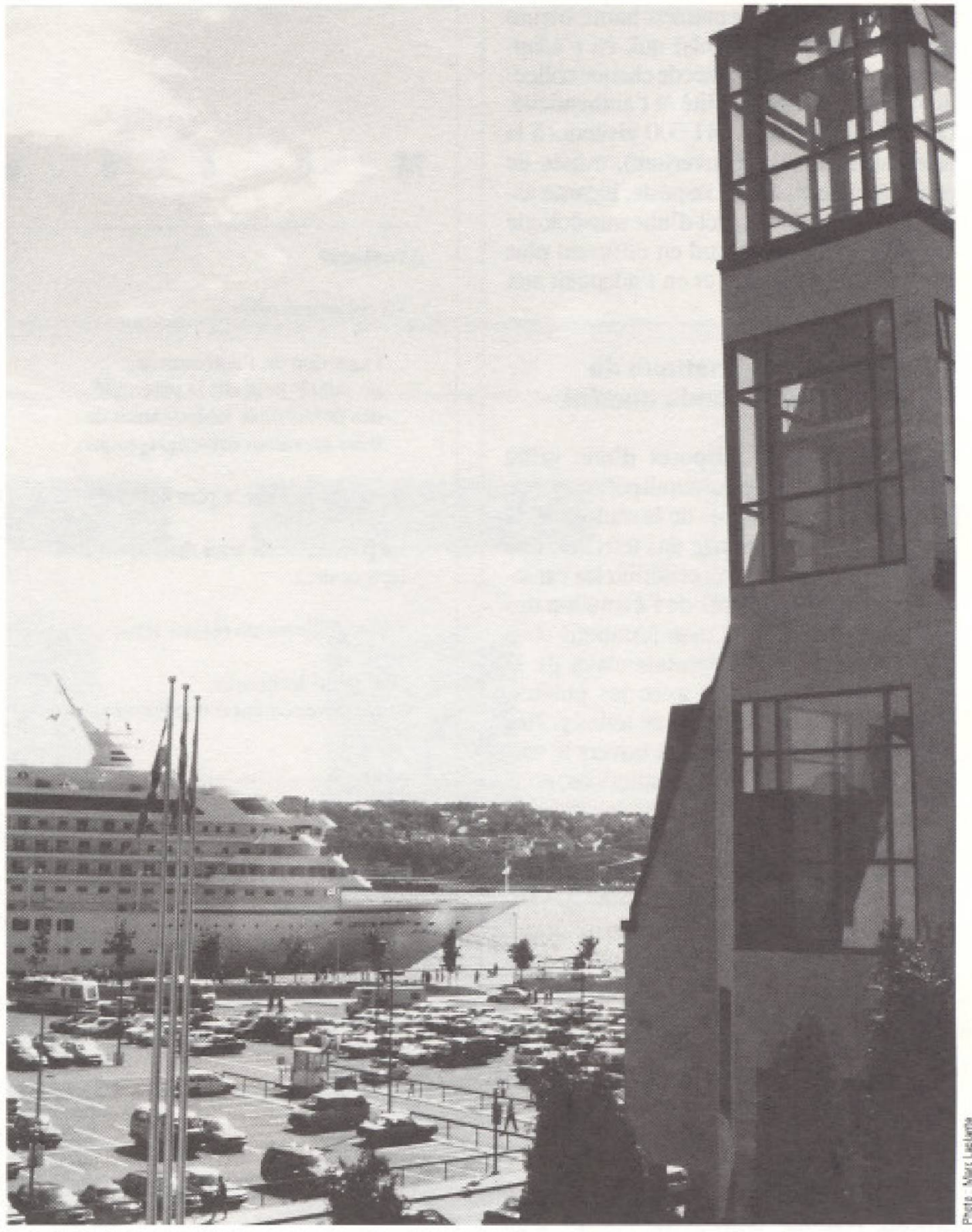

"Les instintions muséles dans leur ensemble constituent un complénent de haur niveau a I'offre touristique dun Elat, dine région ou d'were ville."

mi-faux, etc.) permet de varier le langage visuel des expositions et d'en augmenter a la fois l' intensité et la capacité a exprimer le contenu d'un thème ou d'une collection;

- l'intégration des objets de collection aux expositions scénographiques ( $\mathrm{Fa}$ milles, Mi-vrai/mi-faux, etc.), permet aussi de définir de nouveaux rôles à ces derniers. Leur valeur intrinsèque compose un discoursqui se juxtapose àcelui de leur valeur symbolique ou du discours construit par leur présence à l'intérieur d'un système d'images. Partant, même si le public ne peut technique- ment avoir accès aux significations muscales de ces ajustements, il reste très sensible à leur effet;

- le renouvellement constant des expositions et la multiplication des langages visuels crécent un effet de happening permanent.

Par ailleurs, dans un certain nombre de projets, l'évolution de la réflexion sur l'importance du langage visuel présenté par une exposition et la nécessité d'élargir au maximum l'acces au sens et à la signification des collections (au moins ethnographique) ont permisde créer une formule d'environnement 
intégré (utilisantd'une manière harmonieuse différents médium visuels) qui, en s'adaptantau caractère particulier dechaque collection, en révèle l'originalité et l'authenticité. Le Pare de Miguasha (41 000 visiteurs à la première année de réouverture), musée de paléontologie situé en Gaspésie, incarne assez bien ce nouvel aspect d"une muséologie qui se renouvelle d'abord en diffusant plus largement son contenu et en l'adaptant aux publics.

\section{L'évaluation schématique de I'attractivité du monde muséal}

On aurait souhaitế disposer d'une grille d'analyse scientifiquequiauraitpermis, grâce à l'examen systématique de la multitude de projets nés depuis quinze ans (environ une cinquantaine), de repérer et définir les caractères spécifiques et actifs de l'évolution des musées à travers leurs manifestations concrètes, pour tirer des constats clairs de la relation de ces derniers avec les publics. Mais à notre connaissance, de telles grilles n'existent pas. Néanmoins, à travers le survol sommaire de certaines institutions, nous pouvons tenter de cerner d'une façon schématique les éléments d'attractivité de l'institution muséale en général. Notre exercice a pris en considération les institutions suivantes: les trente musées accrédités du ministère des Affaires culturelles; seize centres d'interpretation historiques de Parcs Canada (en excluant Pointe-au-Pere, Grosse-Ile et les Fortifications de Québec); neuf projets d'interprétation financés conjointement par divers intervenants à titre d'altrait touristique en Gaspésie.

Afin de favoriser une vue analy tique la plus efficace possible, nous avons résumé sous forme de tableau-synthèse les principales caractéristiques que $l^{*}$ on retrouve habituellement dans chaque type générique d'établissement, tout en tenant compte sommairement de l'évolution des dernières années qui tend à une certaine synthèse des genres dans le milieu muséal.

\section{Musée}

A l'heure actuelle, une institution comme le Musée de la civilisation représente une voie originale et prometteuse de mise en valeur puisqu'elle utilise les avantages de la muséologie traditionnelle et de l'interprétation en éliminant beaucoup de leurs irritants. D'autres institutions comme le Parc de Miguasha, le Centre culturel Micmac à Restigouche, le Muscée acadien à Bon-

M

u s é

e

\section{Avantages}

- La collection offre:

- l'émotion de l'authenticité:

. un intérêt basé sur la pérennité: des possibilités intéressantes de mise en valeur musćographique.

- La crédibilité sur le plan culturel.

- La possibilité de tenir des expositions temporaires.

- L'intégration à un réseau, done:

support technique;

renouvellement d'expositions.
Inconvénients

- L'obstacle constitué par l'image publi= que: lieu froid, intellectuel et exclusif à une minorité.

- Le conservatisme encore souventprésent dans la présentation des expositions.

- Encore peu d'accent mis sur la pédagogie dans le parcours.

- L'environnement (aménagement paysager) du bâtiment en région fail souvent défaut de mâme que les services connexes (restauration, pique-nique etc.).

\section{C $e n t r e$ d'interprét ation}

\section{Avantages}

- L'objectif avoué de rejoindre les plus larges publics.

- Le dynamisme des présentations.

- L'intérêt immédiat et l'interaction sont privilégiés.

- Large diffusion d'informations.

- Prise en compte systématique de l'intérét des publics dans un contexte pédagogique.

- Au niveau des sites institutionnels (Parcs Canada), qualité de l'aménagement paysager et des services connexes offerts.

\section{Inconvénients}

- La pédagogie représente la seule forme de prise de contact avec le sujet.

- Désuétude rapide (design) de l'exposition permanente et généralement pas d'exposition temporaire.

- La priorité à la pédagogie crée un abus de l'usage du même médium et une répétitivité des parcours visuels.

- Les sites se distinguent mal les uns des autres.

- Pas de rayonnement ni de crédibilitét établie sur le plan culturel.

- Réseau moins bien structuré que celui des musées. 
aventure, etc , diffusent leurs collections ou leurscontenusen s'occupant del'adaptabilité (a la fois au contenu et aux publics) du langage visuel qu'ils tiennent. Ils échappent aux définitions mais collent davantage aux réalites du public. Le success probable (puisque ces sites, notamment, n'ont que deux années d'existencesur le plan régional) est également fort intéressant.

\section{Constats}

- L'éclosion prévue au cours des prochaines années d'une dizaine de projets axés sur l'adaptabilité des langages visuels pourrait confirmer une tendance des institutions muséales à s' identifier moins par rapport à une école de pensée (interprétation, muséologie traditionnelle, nouvelle musćologic, etc.) que par la qualité de leurs expositions et de leur contact avec les différents publics. Le caractère sacré du musée devrait alors davantage faire place à la diffusion de son contenu et à la signification de son importance.

- En termes de macro-évaluation (analyse des flux touristiques), les centres d'interprétation n'ont pas connu la hausse de fréquentation qui a été celle des musées au cours des dix dernicres années. En général, on peut tracer le portrait de trois types de site:

les sites à forte fréquentation: de 35000 à 140000 visiteurs. Ces centres sont institutionnels (Parcs Canada) et se caractérisent par un aménagement paysager d'envergure (vue sur un plan d'eau, accès à la baignade, etc-) et des installations de pique-nique:

les sites à fréquentation moyenne: de 15000 à 35000 visiteurs. Ces centres (institutionnels également) sont presque exclusivement situes dans des lieux historiques réaménagés (site fortifié par exemple);

les sites à faible fréquentation: 15 000 visiteurs et moins. Ces centres ne comptent que sur leurexposition (doncsur l'attraitdel'interprétation proprement dite) pour attirer le public. Ce sont presque exclusivement les sites aménagés à titre d"attrait touristique.

- On distingue mal, à travers cela, quelle est la motivation premiere du visiteur face à un centre d'interprétation. Fait à souligner, le public qui visite les expositions d'interprétation (et non pas que les sites) présente un profil plus scolarisé que la moyenne.

- Par contraste, la fréquentation des musces traditionnels souleve de l'interêt parce que la motivation de la visite (compte tenudes equipements en place) ne peut être que l'exposition.

\section{L'évaluation de la mise en marché touristique des institutions culturelles}

\section{Diagnostic sommaire sur la commercialisation actuelle du produit culturel}

Dans l'ensemble, en considérant les types génériques muséaux comme un seulet même produit à la fois culturel et touristique, sa commercialisation présente des lacunes à l'heure actuelle.

En ce qui concerne l'interprétation, le produit n'est pas diversifié au niveau des parcours visuels et ne met pas en valeur l'originalité des sites. Par ailleurs, la visite d'une exposition d'interprétation ne semble pas constituer le motif premier de visite d'un centre. Dans ce sens, les musces sont davantage favorisés parce que, règle générale, leurs collections les distinguent déja les uns des autres et donnent à chacun un cachet particulier.

Paradoxalement, les centres d'interprétation disposent $\mathrm{d}^{\prime}$ une approche dynamique que n'ont pas les musés mais ces derniers possèdent une authenticite que n'ont pas les centres d'interprétation. Dans l'unet l'autrecas, le potentiel d'attrait est indéniable, mais le defaut commun demeure la réelle capacité à mettre en valeur les atouts d'un thème ou d'une collection de maniere àen faire ressortir à la fois le sens et par là même, le cachet. Le design n'est pas nécessairement le meilleur médium pour vendre le contenu d'un musée ou d'un centre d'interprétation et les sites qui cherchent à renouveler leur exposition devraient d'abord sedoter d'un véritable coffre à outils incluant divers médium d'intervention.

Pour ce qui est du prix d'entrée des musees, il varie en général entre la gratuité pour des institutions municipales ou religicuses, a un tarif maximal de $10 \$$ pour des institutions de plus grande importance. Les groupes de discussion ont indiqué que les tarifs d'entrée qu'ils sont prêts à payer sont liés à la durée de la visite et à la satisfaction de celle-ci. Beaucoup d'entre eux ont signifié que la gratuité était souvent associéc à la médiocrité.

Actuellement, à part les grands equipements de Montréal et de Québec, les institutions muséales travaillent très peu avec les intermédiaires touristiques que sont les agences de voyages et les agents réceptifs (grossistes québécois ou étrangers). Les gestionnaires, par contre, vont solliciter les responsables de groupes de clubs sociaux, de groupes scolairés ou de personnes âgées.

Quant aux actions promotionnelles mences par les musées, la majorité d'entre eux en sont encore à leurs premiers pas. La clientèle visée est souvent mal définie et le médium utîlisé n'est pas toujours le plus efficace. La faiblesse des budgets entraîne souvent les institutions dans une situation qu'elles peuvent difficilement modifier à elles seules; d'où l'importance de travailler en groupe.

\section{Les composantes d'une stratégie efficace de mise en marché}

La première étape de cette stratégie consisterait à établir un diagnostic des forces et des faiblesses du réseau muséal mais dans un contexte touristique. Cette étape exige une analyse de l'offre quant à ses structures d' accueil (accessibilite physique, heures d'ouverture, capacité pour les groupes, etc.). une analyse plus détaillée de la demande touristique et un diagnostic des actions marketing réalisces par les institutions tant au niveau de la promotion (publicite, relations publiques faites auprès des journalistes en tourisme) que de la commercialisation (liens qu'elles ont pu établir avec des réseaux de distribution ou prescripteurs, forfaits, etc.).

La seconde étape devrait identifier des marchés-cibles afin de créer des couples marchéproduit et d'identifier pour chacun un positionnement. Cette segmentation pourrait se faire, d'une part, par la provenance des visiteurs et d'autre part, par le motif de déplacement des touristes et les activites pratiquées lors de leur séjour. Ces positionnements devraient mettre de l'avant les avantages difrérentiels des institutions muséales en regard des attraits touristiques concurrentiels. 
La troisième étape devrait permettre d'élaborer des stratégies de marketing pour mieux intégrer les musées dans la mise en marché du produit touristique québéoois et des régions concernées. A titre d'exemple, des hypotheses pourraient ctre analysées telles que l' implantation d' une carte inter-musées, un réseau de billetterie liê aux bureaux d" information, etc. En tenant comptedu contexte budgetaire de ces institutions, une approche communicationnelle pourrait suggérer des actions précises au niveau de la publicité, du matériel promotionnel et de leurs présences à certains evénements touristiques (foires, bourses).

\section{La concertation à l'honneur}

Afin que les institutions muséales puissent s"intégrer à part entière au réseau des infrastructures touristiques, il serait opportun de créer des liens entre ces institutions et les autres intervenants touristiques du Québec. D'une part, les gestionnaires pourraient, entre eux, ćlaborer un réseau ou une bannière regroupant des institutions intéressées à participer aux actions marketing visant la clientèle touristique du Québec et celle provenantde l'extérieur de la province et, d' autre part, participer aux campagnes de promotion menées par les représentants touristiques de leur région respective.

Compte tenu qu'un produit touristique est composé de plusieurs éléments (attraits, hébergement, transport, accueil), il est primordial que les représentants de chaque composante travaille en concertation avec les autres intervenants. Dans ce secteur, peu d"équipements sont assez importants pour constituer à eux seuls un produit suffisamment attractif pour justifier le déplacement de la clientèlé.

\section{Conclusion: le devenir touristique des institutions culturelles}

Les institutions musćales dans leur ensemble constituent un complément de haut niveau a l'offre touristique d'un État, d'une région ou d'une ville. Elles identifient qualitativement l'évolution à la fois culturelle, économiqueet sociale d'un lieu, signalent son développement et définissent son image de marque.

Depuis leur création, les musées font l'objet d'une fróquentation assidue qui s'est transformee radicalement depuis vingt ans, sortant de l'exclusivité d"une élite cultivée pour atteindre un cercle de population sans cesse plus large. En cela, la fréquentation nouvelle des institutions témoigne moins d'un engouement particulier de la part du public que

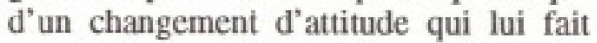
progressivement délaisser les sports pour la culture. Un changement dont les musées ne tirent ni plus ni moins que leur juste part pour I"instant. Cependant, dans une période de changements constants, les témoignages artistique, scientifique, ethnographique ou historique qu'apportent les musces dans la société constituent une source importante de motivations supplémentaires à la visite, qu'il convient d'exploiter.

D'abord, en mettant en valeur les atouts de chaque institution. Avant le savoir, le public qui visite les musées privilégie surtout $l^{\prime}$ emotion. Parmi les non-visiteurs de musceset les visiteurs occasionnels, les groupes de discussion démontrent que ces publics sont d'abord concernés par le caractère authentique et original d'un lieu et $d^{*} u n e$ collection ce qui, dans un deuxième temps, devient un levier pour l'apprentissage. Globalement (sauf pour les musées scientifiques et techniques), le lieu muséal constitue par nature un produit à caractère affectif et l'information n'est pas nécessairement la motivation première de sa fréquentation.

Dans ce contexte, le musée doit ensuite tabler sur une mise en valeur adéquate de ses atouts. Identifier le musée auprès des visiteurs et en signifier le sens d'une façon manifeste sur le plan visuel constituent à ce niveau des priorites. Essentiellement, c'est la présentation des expositions qui incarne l'originalité du lieu et exprime son sens et son contenu pour le visiteur. Afin d"adapter son processus de diffusion culturelle auprès du plus large public et faire de son contenu un motif évident d'attrait, le milieu muséal devra donc voir au cours des prochaines annés à se doter d'un véritable coffre à outils museographique (scénographie, scénographie d'art, environnement intégré, etc.), ainsi que des compétences ad hoc pour en assurer le bon usage; il echappera ainsi à l'uniformisation stylistique consécutif à l'usage d'un seul médium.

Les musées qui maîtrisent bien l'aspect théorique du caractere unique et de l'importance de leurs themes et collections doivent désormais le transposer dans le champ de la pratique et s'assurer de contrôler les langages visuels qui assurent l'acees de chaque visiteurą l'institution et l'attractivitédecelleci.
Enfin, la création d'un réseau" et la promotion constituent les instruments indispensables dont devront se doter les musées pour maximiser les retombees de leur positionnement actuel sur l'échiquier touristique. -4

"N.D.L.R. : On lira p.39et 40, l'article de Svivie Gagnon a ce sulet.

\section{NOTES ET RÉFÉRENICES}

(1) Marylin Gilbertson Hood, Adults Attitudes Toward Lalsure Choices in Relation to Musoum Participation, mémoire de l'Université de I'Ohis, 1961.

(2) Jean Davalon et Hana Gottesdiener. Représentations of attentes des visiteurs du Musébe national des techniques, Lyon, fovrier 1992

[3] Gilles Pronovost, Les comportements das Québucois an matiàre d'activitess culturelles de loisir, Les publications du Quebec, 1989.

(4) Ministèredes Affairesculturelles, Tableaux d'alde au calcul des subventions aux institutions muséales. 1990.

(5) Groupe SECOF, Etudo sur la robalité economique des musbes quŚbibcols, 1992

\section{PERSONNES ET OUVRAGES CONSULTES}

CANADIAN BUSINESS ART COUNCIL, Rapport d'enquảto 1990

GOTESDIENEF, Hana, Freins et motivations a la visite des musées d'art, ministère de la Culture, Departement des Etudes et de la Prospective. France, mai 1992

IDEATION COMMUNICATIONS; ZINS, BEAUCHESNE ETASSOCIES, FOCUS-GAOUPES, Musde du cuir de Lor etteville, stude de faisabilite 1990; Musé do Findustria laitiore, btude do faisabilití 1991.

SERVICE CANADIEN DES PARCS. Rapport statistique 1988, rógion de Qub́bec.

SERVICE CANADIEN DES PARCS, Rapport statis tique 1990, région de Oubbec.

STATISTIQUES CANADA, Division de l'education, de la culture et du tourisme. Enqubte sur los atablissements du patrimoine, 1989-1990.

UZZEL, David, "Les approches socio-cognitives de Fevaluation des expositions ". Publics et Museres. no 1, Lyon, Editions des Presses Universitaires de Lyon, mai 1992.

Entrevue avec Philippe Mairot, président de la Fédera. tion des Ecomustes de France, juin 1992

Entrevue avec Cécile Ouellet, chargée de projet, Musée de la civilisation, Qubbec, juin 1992 